\title{
An a-Formylglycine Building Block For Fmoc-Based Solid-Phase Peptide Synthesis
}

\author{
Jason Rush $\ddagger$ and Carolyn R. Bertozzi ${ }^{\star}, \ddagger, \dagger$ \\ Department of Chemistry, Department of Molecular and Cell Biology, and Howard Hughes Medical \\ Institute, University of California, Berkeley, CA 94720
}

Abstract

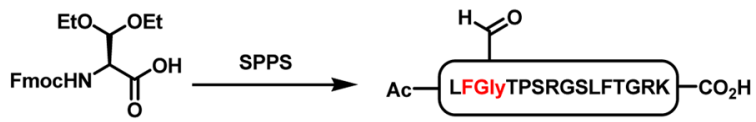

Nearly all known sulfatases share a common active site modification that is required for their activity: conversion of cysteine to $\alpha$-formylglycine. We report the synthesis of an $\alpha$-formylglycine building block suitable for Fmoc-based solid-phase peptide synthesis. The building block was incorporated into a synthetic peptide derived from the active site of a Mycobacterium tuberculosis sulfatase.

Sulfation of biomolecules is a common modification that is involved in both normal and pathological processes. ${ }^{1}$ For example, sulfated oligosaccharides play important roles in inflammation, 2 tyrosine sulfation is linked to the recognition of target cells by HIV $-1,3$ and an abundance of sulfated glycolipids is correlated with Mycobacterium tuberculosis strain virulence. ${ }^{4}$

Two classes of enzymes control sulfation. Sulfotransferases catalyze the formation of sulfate esters, ${ }^{5}$ while sulfatases catalyze their hydrolysis. ${ }^{6}$ Sulfatases utilize a unique mechanism that is facilitated by an unusual residue in the active site, $\alpha$-formylglycine (FGly) (1) (Figure 1). In the proposed mechanism, FGly is hydrated in the active site ${ }^{7}$ and the geminal diol (2) acts as a nucleophile to produce a sulfated enzyme intermediate (3) (Figure 1). This intermediate then collapses, expelling inorganic sulfate and regenerating FGly.

FGly is installed in the sulfatase active site via a post-translational modification prior to folding. 8 A genetically-encoded cysteine residue is oxidized to form FGly by a recently characterized enzyme termed the formylglycine generating enzyme, or FGE. ${ }^{9}$ FGE recognizes a consensus sequence around the target cysteine that is highly conserved across all species and is found in all sulfatases. ${ }^{6}$ Interestingly, some bacterial sulfatases employ a serine residue in place of cysteine while retaining the overall consensus sequence. These sulfatases are modified by a distinct mechanism. 10

Biochemical studies of sulfatases and FGEs could benefit tremendously from the availability of synthetic peptides bearing the central FGly residue. Currently, one scalemic synthesis of FGly has been reported, which utilizes the highly versatile Williams' glycine template. ${ }^{11}$ However, an Fmoc-protected variant has not been reported and FGly analogs have not been incorporated into synthetic peptides. Here we report an efficient synthesis of a Fmoc-protected

\footnotetext{
crb@berkeley.edu.

Department of Chemistry

$\dagger$ Department of Molecular and Cell Biology

*Howard Hughes Medical Institute
} 
FGly building block and its incorporation into a synthetic peptide derived from a Mycobacterium tuberculosis sulfatase. The procedure requires no chromatography and produces Fmoc-FGly in 6 steps in $>70 \%$ overall yield.

In designing a FGly analog suitable for solid-phase peptide synthesis (SPPS), we chose to protect the aldehyde functionality as an acetal that could be removed alongside other side chain protecting groups during the acidic cleavage step of Fmoc-based SPPS. The diethyl variant was utilized as both literature precedent 12 and our own experience indicated that the dimethyl acetal was too resistant to acidic cleavage. The synthesis began with protection of commercially-available D-Ser-Ome-HCl using Fmoc-OSu to provide Fmoc-protected amino acid ester $4^{13,14}$ in nearly quantitative yield (Scheme 1 ). Exposure to catalytic $\mathrm{BF}_{3} \cdot \mathrm{Et}_{2} \mathrm{O}$ and 2,2-dimethoxypropane afforded oxazolidine $\mathbf{5}$ in excellent yield. ${ }^{15}$ Sequential reduction and TEMPO-mediated oxidation ${ }^{16}$ yielded an Fmoc variant of Garner's aldehyde (6) in $92 \%$ yield over two steps. $\mathrm{LiBH}_{4}{ }^{17}$ is the optimal reductant in this sequence, as other conditions (i.e., DIBAL-H, $\mathrm{NaBH}_{4}$ in protic media, $\mathrm{LiAlH}_{4}$ ) partially cleaved the Fmoc group. Simultaneous protection of the aldehyde and ring opening were effected in refluxing EtOH with catalytic CSA to furnish amino alcohol 7. Finally, TEMPO-mediated oxidation ${ }^{18}$ of the primary alcohol provided the desired SPPS building block $\mathbf{8}$ in high yield. Notably, all intermediates were either taken on crude or purified via recrystallization.

With building block 8 (Fmoc-FGly $\left.(\mathrm{OEt})_{2}-\mathrm{OH}\right)$ in hand, we sought to determine its enantiomeric purity and coupling efficiency for incorporation into peptides. Thus, the FGly building block was coupled to both chiral and racemic $\alpha$-methylbenzylamine using EDC/HOBt. ${ }^{19}$ Following extractive workup, ${ }^{1} \mathrm{H}-\mathrm{NMR}$ analysis of the products formed from the chiral amine indicated that a single stereoisomer was formed. Analysis of the reaction containing racemic amine indicated two diasteriomers were formed in approximately equal amounts. ${ }^{19}$ These results suggest that the coupling reaction proceeds without racemization at the $\alpha$-carbon, and that the starting material was $\geq 95 \%$ enantiomerically pure.

With the coupling reaction characterized, we synthesized the consensus sequence derived from a Mycobacterium tuberculosis sulfatase, tetradecapeptide 9 (LFGlyTPSRGSLFTGRK). Given the sensitive nature of the aldehyde functionality, we expected that standard peptide cleavage cocktails containing silanes or thiols would not be compatible. Indeed, exposure of resin-bound model peptides to cleavage cocktails containing silanes (i.e., TIS, TES) resulted in reduction of FGly to serine, while addition of ethanedithiol resulted in quantitative formation of the dithioacetal. Fortunately, thioanisole and anisole were found to be satisfactory alternative scavengers. In addition to these anticipated issues, an unexpected problem arose when the fulllength peptide was cleaved, namely observation of an abundant byproduct ( $\sim 50 \%$ by HPLC peak integration) with a mass $172 \mathrm{Da}$ greater than the desired product. The side reaction occurred independently of the cleavage cocktail and the abundance of the byproduct increased as a function of time. Screening of model tripeptides indicated that the reaction was associated with the Fmoc- $\operatorname{Arg}(\mathrm{Pbf})-\mathrm{OH}$ building block. Purification of the byproduct formed from a model tripeptide (Ac-Arg-FGly-Ala-OH) allowed its structure to be assigned as $\mathbf{1 0}$ (Figure 2) by HRMS and 2D NMR. ${ }^{19}$

This unique structure likely results from a Friedel-Crafts-type reaction between the cleaved 2,2,4,6,7-pentamethyldihydrobenzofuran-5-sulfonyl ( $\mathrm{Pbf}$ ) protecting group and FGly (Figure 3). The initial attack of the electron rich aromatic ring (11) on the FGly aldehyde could occur either before or after desulfonation to form transient intermediate 12. Loss of either a proton or $\mathrm{SO}_{3}$ would provide 13, an adduct we detected by LCMS analysis. Subsequent dehydration then results in the proposed modified peptide $\mathbf{1 4}$. 
This was an interesting development as most side reactions during peptide cleavage result from the nucleophilic nature of amino acid side chains. These are avoided by the inclusion of nucleophilic scavengers in the cleavage reaction (i.e., silanes, EDT, anisole). However, the electrophilic nature of the FGly side chain produces a novel situation in which the scavengers have either no effect, or worse, react with the FGly residue. We attempted to avoid the side reaction during peptide cleavage using (1) various nucleophilic scavengers, (2) altered cleavage temperatures, or (3) electrophilic scavengers (i.e., aromatic aldehydes, 1,3 dicarbonyl containing compounds). None of these conditions suppressed formation of the byproduct. We therefore conclude that the Pbf protecting group is incompatible with FGly.

Accordingly, an alternative arginine building block was employed. Using commercially available Fmoc- $\operatorname{Arg}(\mathrm{Boc})_{2}-\mathrm{OH}$, the synthesis and cleavage of tetradecapeptide 9 was accomplished without incident (Figure 4). Exposure of purified peptide to $\mathrm{MeONH}_{2}$ afforded complete conversion of the aldehyde to oxime 15 (Figure 5), confirming that the aldehyde retains normal reactivity.

In summary, the Fmoc-FGly building block and SPPS methods for its use that we report here should facilitate studies of sulfatases and FGEs that activate them. In addition, the ability to synthesize peptides with internal electrophilic groups such as the aldehyde may find use in generating cyclic peptides or in conjugating unnatural epitopes to peptides for biological studies.

\section{Supplementary Material}

Refer to Web version on PubMed Central for supplementary material.

\section{Acknowledgements}

We thank Fiona Lin for NMR assistance. This work was supported by a grant to C.R.B. from the National Institute of Health (AI51622).

\section{References}

1. Hemmerich S, Verdugo D, Rath VL. Drug Discov Today 2004;9:967-975. [PubMed: 15539140]

2. Rosen SD. Am J Pathol 1999;155:1013-1020. [PubMed: 10514381]

3. Farzan M, Mirzabekov T, Kolchinsky P, Wyatt R, Cayabyab M, Gerard NP, Gerard C, Sodroski J, Choe H. Cell 1999;96:667-676. [PubMed: 10089882]

4. Goren MB, Brokl O, Schaefer WB. Infect Immun 1974;9:150-158. [PubMed: 4271720]

5. Chapman E, Best MD, Hanson SR, Wong CH. Angew Chem Int Ed 2004;43:3526-3548.

6. Hanson SR, Best MD, Wong CH. Angew Chem Int Ed 2004;43:5736-5763.

7. Boltes I, Czapinska H, Kahnert A, von Bulow R, Dierks T, Schmidt B, von Figura K, Kertesz MA, Uson I. Structure 2001;9:483-491. [PubMed: 11435113]

8. Dierks T, Schmidt B, von Figura K. Proc Natl Acad Sci U S A 1997;94:11963-11968. [PubMed: 9342345]

9. Preusser-Kunze A, Mariappan M, Schmidt B, Gande SL, Mutenda K, Wenzel D, von Figura K, Dierks T. J Biol Chem 2005;280:14900-14910. [PubMed: 15657036]

10. Szameit C, Miech C, Balleininger M, Schmidt B, von Figura K, Dierks T. J Biol Chem 1999;274:15375-15381. [PubMed: 10336424]

11. DeMong DE, Williams RM. Tetrahedon Letters 2002;43:2355-2357.

12. DeMong DE, Williams RM. J Am Chem Soc 2003;125:8561-8565. [PubMed: 12848564]

13. While the $\mathrm{L}$ isomer of 4 is commercially available and has been made synthetically (see ref. 14), for cost effeciency and safety we adopted another route.

14. Gomez-Vidal JA, Forrester MT, Silverman RB. Org Lett 2001;3:2477-2479. [PubMed: 11483039] 
15. McKillop A, Taylor RJK, Watson RJ, Lewis N. Synthesis 1994:31-33.

16. Janusz J, Gryko D, Kobrzycka E, Gruza H, Prokopowicz P. Tetrahedon 1998;54:6051-6064.

17. Brown HC, Narasimhan S, Choi YM. J Org Chem 1982;47:4702-4708.

18. Zhao M, Li J, Mano E, Song Z, Tschaen D, Grabowski EJJ, Reider P. J Org Chem 1999;64:25642566.

19. See Supporting information. 


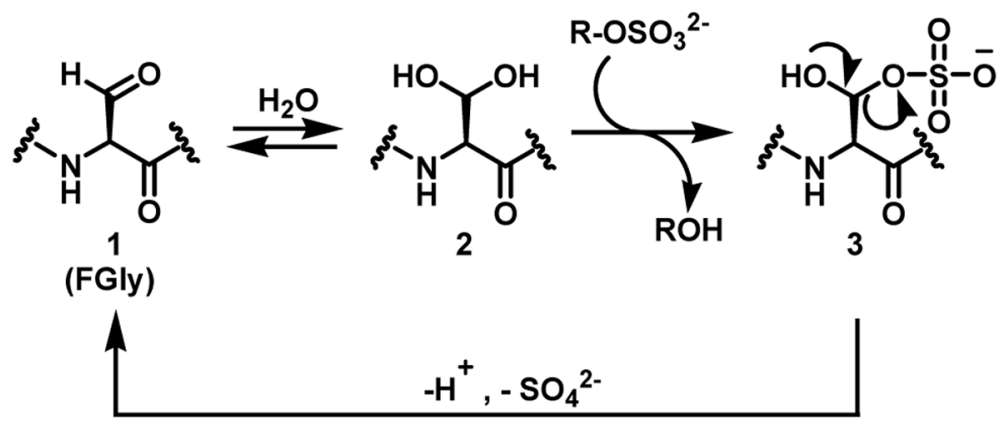

Figure 1.

Catalytic cycle of sulfatases. 


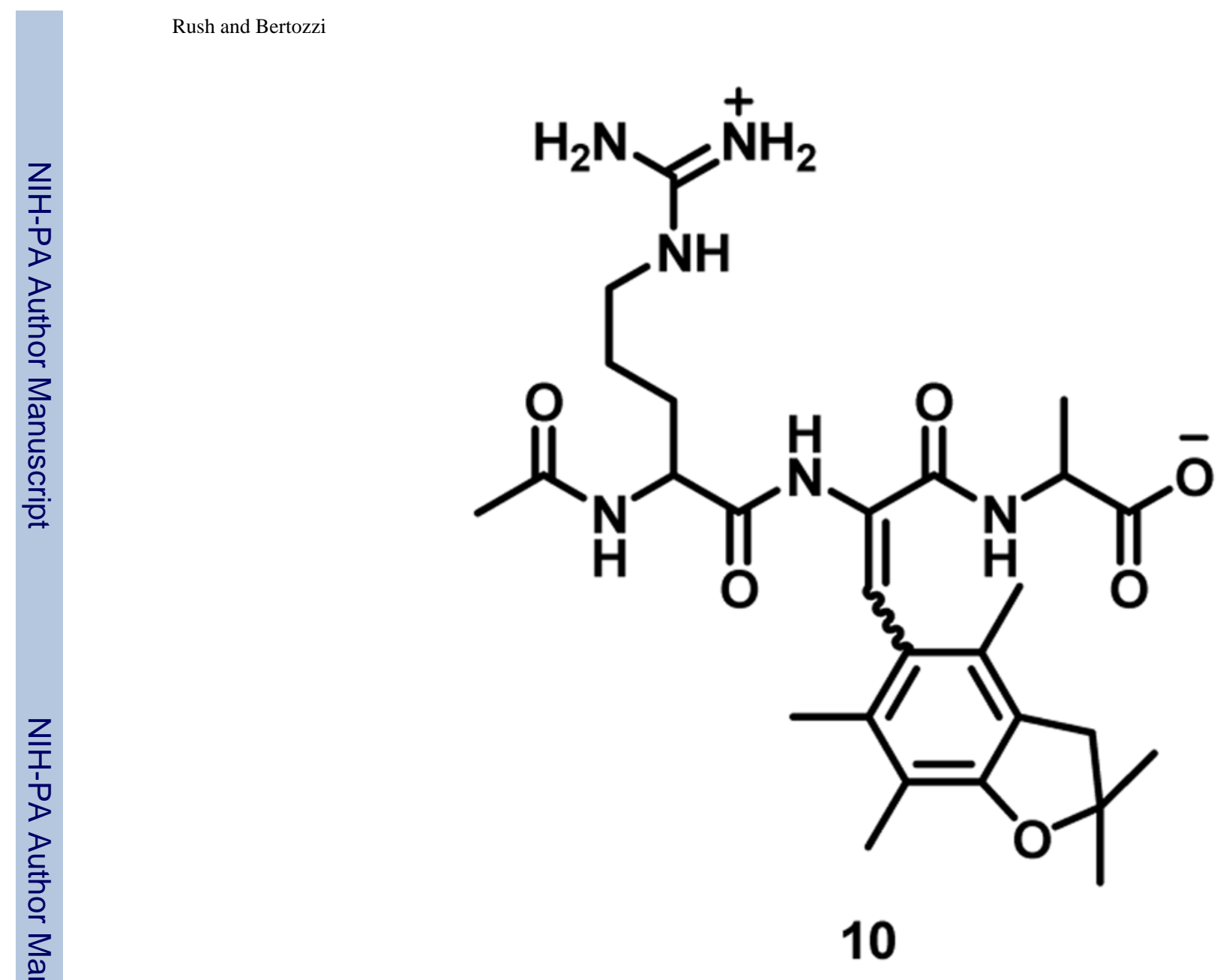

Figure 2.

Modified tripeptide $\mathbf{1 0 .}$ 


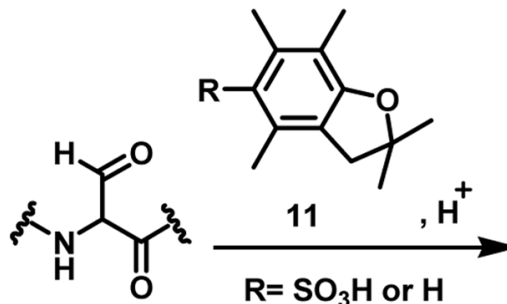<smiles>[Y]C(=O)C(NC(C)(C)C)C(O)C([R])(C(C)=O)C1=C(C)C(C)=C(C)C2=[O+]C(C)(C)CC21</smiles>

12

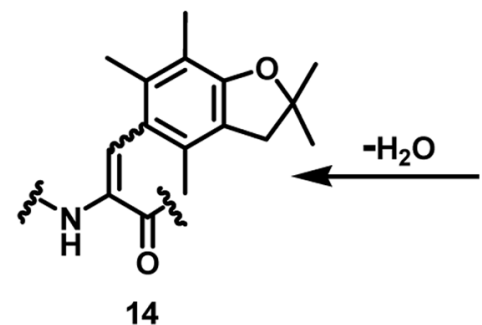

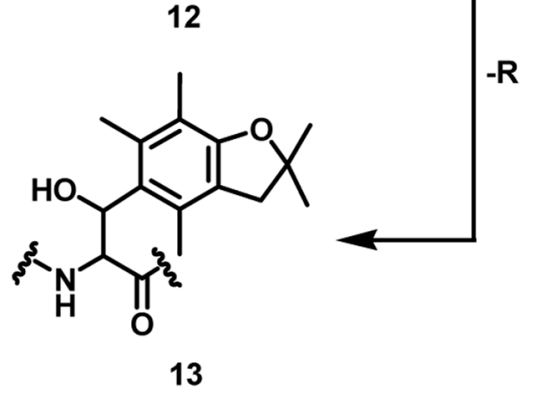

Figure 3.

Proposed mechanism for the formation of byproduct. 
773.7

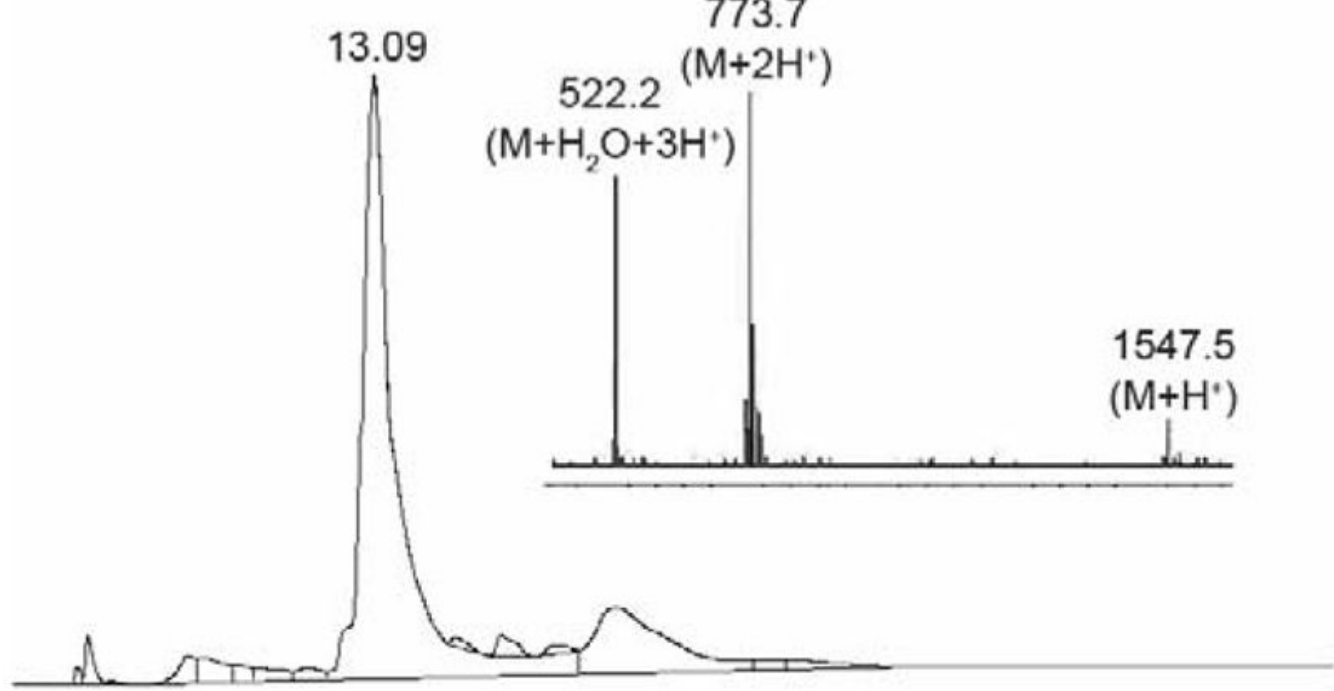

10

20

30

40

Figure 4.

HPLC and mass spectrometry analysis of $\mathbf{9}$. 


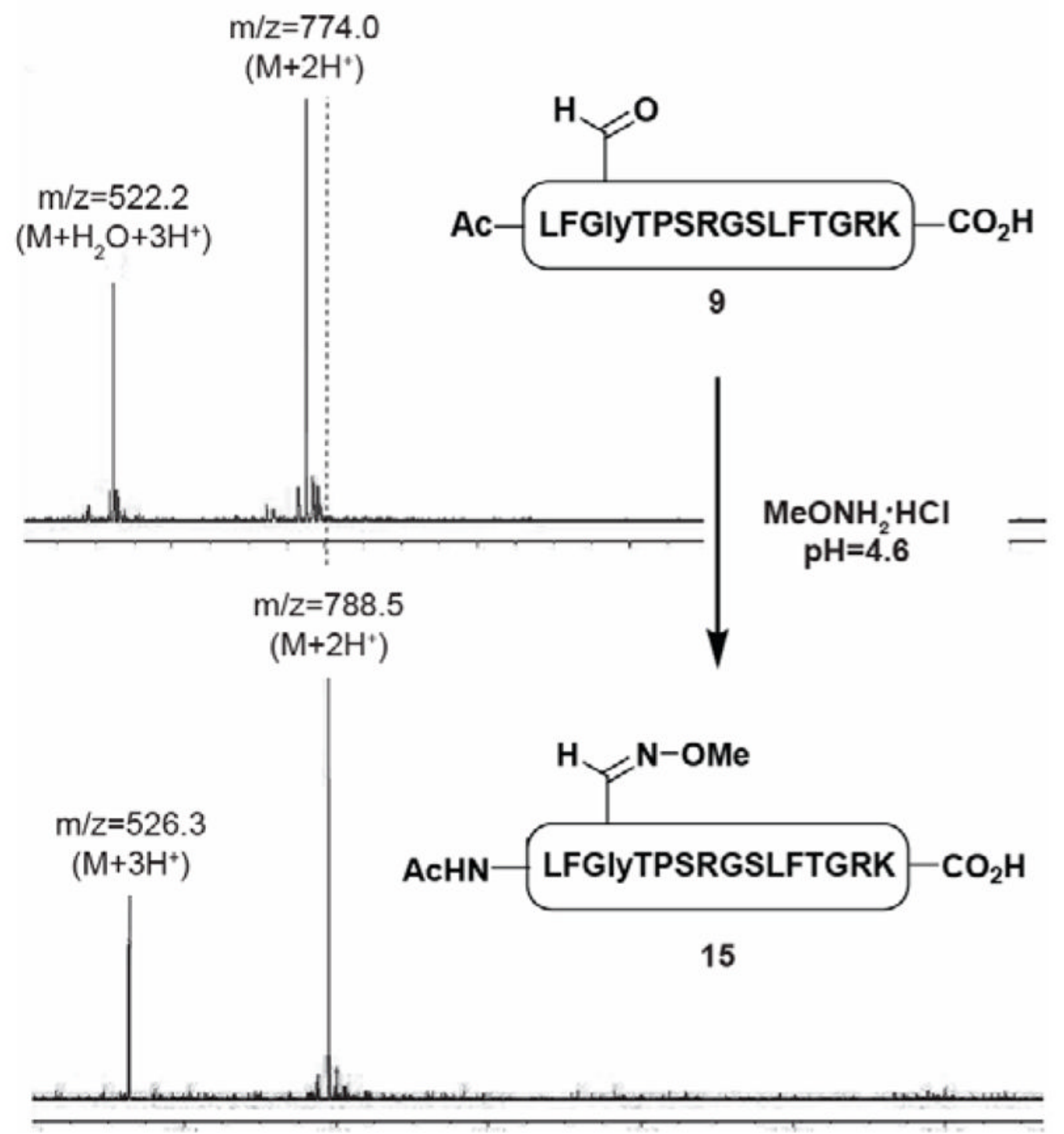

Figure 5.

Mass spectrometry analysis of peptide $\mathbf{9}$ and its oxime adduct $\mathbf{1 5}$. 


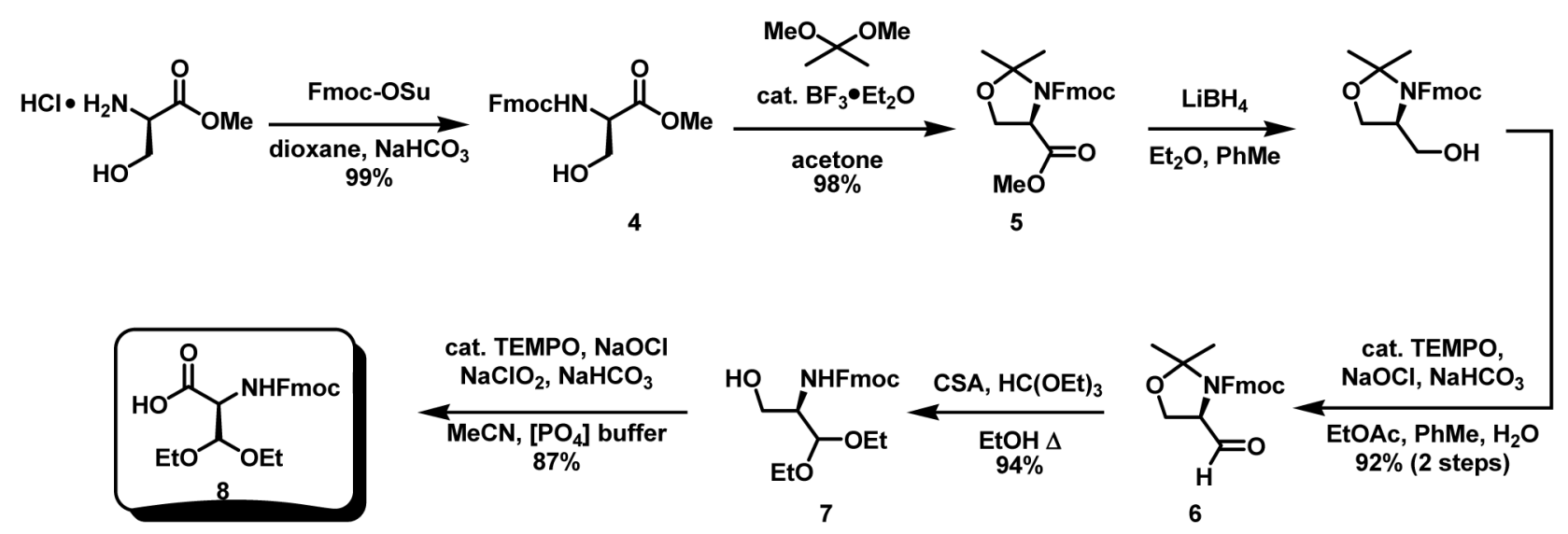

Scheme 1.

Synthesis of Fmoc-FGly Building-Block 8 